Session 1238

\title{
Distance Collaborations With Industry
}

\author{
A. Peskin, K. Swyler \\ Brookhaven National Laboratory
}

\section{The Case for Distance Collaborations}

The college-industry relationship has been identified as a key policy issue in Engineering Education. [1] Collaborations between academic institutions and the industrial sector have a long history and a bright future. For Engineering and Engineering Technology programs in particular, industry has played a crucial role in many areas including advisement, financial support, and practical training of both faculty and students. Among the most important and intimate interactions are collaborative projects and formal cooperative education arrangements.

Most recently, such collaborations have taken on a new dimension, as advances in technology have made possible meaningful technical collaboration at a distance. [2] There are several obvious technology areas that have contributed significantly to this trend. Foremost is the ubiquitous presence of the Internet. Perhaps almost as important are advances in computer based imaging. Because visual images offer a compelling user experience, it affords greater knowledge transfer efficiency than other modes of delivery. Furthermore, the quality of the image appears to have a strongly correlated effect on insight. A good visualization facility offers both a means for communication and a shared information space for the subjects, which are among the essential features of both peer collaboration and distance learning. [3]

\section{Enabling Infrastructure}

In order to be capable of participating in a visualization-based distance collaboration, a number of infrastructure elements need to be in place at the participating institutions. First, one needs a robust network connection. There are few technically interesting data files smaller than 10 megabytes (MB); many are in the range of gigabytes. So at a minimum, to achieve a reasonably interactive facility, a connection in the range of T1 (1.5 Megabits per second) to T3 (44 megabits per second) would appear necessary. The availability of bandwidth of up to DS3 (155 Mbps) to the post secondary community through the Internet 2 program portends that bandwidth in that range will soon be the norm. Achieving this level of connectivity may be one of the greatest challenges for Engineering Technology schools, many of which are geographically isolated and hard 
pressed to make the financial investment represented by subscriptions to this level of service.

Computer platforms are somewhat less of a problem owing to the truly amazing advances in speed, capability and cost effectiveness of desktop units. For less complex files and two dimensional images, modern commodity PCs with multimedia capability often suffice, while for larger data sets and stereographic reproduction, graphics-enabled multipipe workstations would be necessary for satisfactory results.

Ultimately, presentation of high-quality images rests with the projection equipment. High resolution and brightness are clearly necessary ingredients for a realistic effect. For stereography, two distinct images must be presented to the viewer, one for each eye. There are two common methods of image separation, a time shared screen synchronized with shuttered goggles, or the use of polarizing filters on each of two projectors' lenses coupled with similarly polarized viewing glasses. Enhancements such as head position sensors and animation further enrich the possibilities for meaningful analysis, not to mention their entertainment value which tends to increase the viewers' attention span.

There are important human factors to be considered at both the origin and viewer ends of the chain to make for a rewarding viewing experience. At the origin, one must pay heed to production values; for example, if a lecture is being transmitted, the lecturer should be trained to make eye contact with the camera, lighting should be appropriate, and so forth. At the viewer facility, conditions should be conducive to learning and should give the appearance of immediacy, such as one might find in a conference room setting. There must be sufficient technology in place to achieve interactivity, whether it be a channel for allowing the viewer to ask the lecturer a question, or the ability for collaborators at more than one location to virtually manipulate an image.

\section{Technical Applications}

In order to be suitable for Engineering Technology education, technical applications must meet several criteria. They must be highly visual in nature, pedagogical, interactive, and based on real science or engineering problems. There are a growing number of applications that fall into that category.

Mechanical CADCAM is a prime example. A stereographic rendering of a drawing that can be zoomed, rotated, translated, sliced, and navigated, all before it is fabricated, makes a strong positive impression on its designers. This is particularly true if they are students.

Medical imaging represents another example. CAT scans and MRI techniques are now commonplace, and distance diagnosis is becoming a reality. Stereo 3D is particularly useful in dosage monitoring, where the problem is to apply the treatment where the pathology is, while avoiding exposing healthy tissue to its possibly toxic effects. Images in two modalities, one showing the anatomy and a malignant tumor, for instance, and another showing dosage distribution, can be superimposed on each other for accurate treatment planning. 
Environmental technology is another area that benefits greatly from visualization techniques. The spread of environmental insults can be depicted flowing through the atmosphere, the ground, or the oceans. Comprehension can be enhanced by using animation techniques to trace the progress of the pollutants or its remedies.

There are many other suitable applications, in the fundamental sciences such as physics and chemistry, and in applied areas such as oil exploration and protein crystallography. Use of this facility is also of obvious benefit in teaching computer technology. Collaborative applications also provide lessons in teamwork, leadership, and responsibility in ways that conventional course delivery cannot. Perhaps such a facility's greatest advantage is in showcasing cross-disciplinary activities, where one may find, for example, chemical, medical, and computer technologists working on different aspects of the same problem, with each gaining insight and respect for the work of the others.

\section{A Coordinated Program}

Brookhaven National Laboratory (BNL) is a multi-disciplinary research laboratory of the U. S. Department of Energy. It is the home for thousands of scientists, engineers and technicians. Among its world-class scientific instruments are the National Synchrotron Light Source, the Alternating Gradient Synchrotron, the High Flux Beam Reactor, and the Relativistic Heavy Ion Collider currently in development. It is also the base for unique information repositories such as the Protein Data Bank and the National Neutron Data Center which are referenced daily by hundreds of researchers all over the world.

BNL has also long had a vibrant education program reaching out to faculty and students at every academic level. Undergraduate research participation has been a mainstay of BNL's educational programs since 1952. Summer and academic semester student internships in the Laboratory's research divisions provide training which is available no other way. Similar summer opportunities are available for secondary science, mathematics and technology teachers, as well as in-service courses covering current research topics of special interest. Primary teachers are offered experimental 'science immersion workshops,' intended to help promote inquiry-based learning in the elementary classroom. Student programs support inquiry-based learning at all levels, and reach out to underrepresented groups. In all of these areas, participation may be enhanced and extended through distance collaborations with BNL and others via the Internet.

Recently, discussions between BNL and staff at a number of prominent Engineering Technology institutions has emphasized enriching a vital, yet historically neglected segment of the science and technology education spectrum, the Engineering Technician and Technologist. The overall objective is to enhance both the curricular content and the computing infrastructure of the cooperating institutions and bring them together into a network connected "virtual campus." The ultimate goal can be said to be a continuing program of co-operative education at a distance. [4] 
Stereographic visualization is at the very heart of this concept. The BNL stereo visualization theatre consists of a networked Silicon Graphics Onyx computer coupled to two Barco high-intensity projectors, to which polarizing filters have been fitted. The images are back-projected onto a special fresnel effect screen that preserves light polarization. The images are produced in a conference room setting with a remarkable degree of realism. This facility was first developed for a collaborative program with Mobil Oil to evaluate the microscopic properties of oil reservoir rock. It has since been applied to all of the other scientific areas mentioned above. [5] This facility is the prototypical 'classroom' for this project and is designed for ease of replication back at the participants home institutions, where the work may continue.

There are a number of unique aspects to the envisioned program. First, it has both on-site at BNL and home campus segments. Secondly, it involves both the students and the faculty, who are charged with providing the continuity after the participating students graduate. Finally, it addresses not only the individual students' education, but also their home institutions' computing infrastructure and its courseware.

While seeking funding for a full scale implementation of this vision, some steps have already been taken. BNL has established educational partnerships with seven community colleges in the Northeast. Through them, students participate in preparatory inter-term minisemesters, and in academic semester and summer appointments in BNL's technical programs. Semester students receive academic credit for the experience. A new community college faculty-student research program is intended to help the schools develop their own resources in engineering technology and other areas. The Laboratory is also a member of the Northeast Partnership for Environmental Technology Education (NE PETE), an association of community and technical colleges and other organizations focused on providing training for HAZMAT technicians.

\section{Conclusion}

It has long been recognized that quality engineering education should present the student with an environment that duplicates as much as possible that which the graduate will encounter in industry. And there is growing evidence that collaboration with the industrial sector is intensifying; a recent issue of ASEE Prism highlighted several recent notable case histories of collaboration. [6] A recent NSF-sponsored workshop on Engineering Technician Education also urged collaboration of a different kind; that "...collaboration and teaming should be fostered and supported within and between academic communities," as well. [7] The growth of visualization-based distance collaborations in the modern engineering work environment is not a development that educational institutions can long afford to ignore. 


\section{Bibliography}

1. R. Morgan, N. Kannankutty, D. Strickland, Future Directions for University-Based Research, ASEE Prism, March 1997, pp 30-36.

2. R. Kouzes, J.Myers, W. Wulf, Collaboratories: Doing Science on the Internet, IEEE Computer, Volume 29, Number 8, Auguest 1996

3. R. Fielding, G. Kaiser, The Apache HTTP Server Project, IEEE Internet Computing, Volume 1, Number 4, July-August 1997 pp 88-90

4. A. Peskin, J. Curry, R. Denning, J. Waintraub, Engineering Technology Enrichment Through Distance Collaborations, Conference on Industry Education Collaborations, Savannah GA, February 1998

5. A. Peskin, B. Andrews, B. Dowd, K. Jones, P. Siddons, Microtomography with 3-D Visualization, Proceedings of the SPIE Annual Meeting, Denver Co, June 1996

6. ASEE Prism Special Industry Issue, January 1997

7. A National Agenda for the Future of Engineering Technician Education, Workshop Report, Sinclair Community College, Dayton OH, January 1997

\section{Biographical Information}

A. PESKIN - Arnold Peskin is a Senior Scientist and past Head of the Computing and Communications Division at Brookhaven National Laboratory. Mr. Peskin is a Senior Member of the IEEE, a Fellow of the Accreditation Board for Engineering and Technology, and past Chair of ABET's Technology Accreditation Commission. A graduate of New Jersey Institute of Technology with a BSEE and Polytechnic University with an MSEE, he has also served as Adjunct Professor at Columbia University and SUNY at Stony Brook.

K. SWYLER - Karl J. Swyler holds a BS in Physics from Stevens Institute of Technology and a Doctorate in Optics from the University of Rochester. Following his undergraduate training, he was involved in electrostatic imaging R\&D at the Xerox Corporation. After a postdoctoral appointment in Materials Science at SUNY Stony Brook, he joined the scientific staff of Brookhaven National Laboratory in 1973. In 1988 he joined the Laboratory's Office of Educational Programs, and in 1994 was named its Head. Dr. Swyler is a member of Tau Beta Pi, and serves on several educational advisory boards. 